\title{
Efficacy of intra-articular methylprednisolone along with platelet rich plasma vs. platelet rich plasma in early osteoarthritis: a retrospective study
}

\author{
Ketas K. Mahajan', Sneh H. Dudhia ${ }^{2 *}$ \\ ${ }^{1}$ Department of Orthopaedics, ${ }^{2}$ Department of Pharmacology, Parul Institute of Medical Sciences and Research, \\ Vadodara, Gujarat, India
}

Received: 27 July 2021

Revised: 16 September 2021

Accepted: 22 September 2021

*Correspondence:

Dr. Sneh H. Dudhia,

Email: drsneh1011@gmail.com

Copyright: $\odot$ the author(s), publisher and licensee Medip Academy. This is an open-access article distributed under the terms of the Creative Commons Attribution Non-Commercial License, which permits unrestricted non-commercial use, distribution, and reproduction in any medium, provided the original work is properly cited.

\begin{abstract}
Background: Increased prevalence as well as burden affecting osteoarthritis of knee joint has led to increase in concerns regarding safety of various pharmacological methods. Hence, there is an increase in demand for newer and effective strategies for treatment and management. Thus, a novel alternate for treatment is required that might repair damage to cartilage rather than simply reduce related symptoms. The aim of this study was to study the efficacy of intra-articular methylprednisolone along with platelet rich plasma versus platelet rich plasma in early knee osteoarthritis.

Methods: This retrospective study was conducted on 60 patients diagnosed with initial stages of osteoarthritis of knee according to Kellgren-Lawrence classification. Patients age range was between 40 to 80 years who were divided into 2 groups; group I: It contained 30 subjects treated with intra-articular injections of Platelet Rich Plasma in combination with methylprednisolone and group II: comprised of 30 patients treated with intra-articular injection of Platelet rich Plasma alone. Clinical data pertaining to medical history, VAS and KOOS scores were collected. Statistical analysis was performed using the student's $t$ test.

Results: Statistically significant improvements in VAS and KOOS score values were observed on assessment of scores at baseline, 1, 3 and 6 months intervals, respectively.

Conclusions: It can be concluded from the study that a combination therapy of intra-articular injection of combination of PRP with methylprednisolone yields superior results when compared to use of PRP injections alone.
\end{abstract}

Keywords: PRP, Methylprednisolone, Intra-articular, Early osteoarthritis

\section{INTRODUCTION}

Osteoarthritis is a condition which is chronic and noninflammatory origin rheumatological disease affecting the synovial joints. It is characterized with degeneration of joint cartilage, sub-chondral changes in bone and also, synovitis. ${ }^{1}$ Non-steroidal anti-inflammatory agents along with other agents such as glucosamine, chondroitin sulfate, hyaluronic acid etc have been generally used for reduction of inflammatory process thereby, causing pain relief in patients suffering from osteoarthritis. These agents have shown limited results in causing reduction in degeneration of chondrocytes thereby, causing an improvement in cartilage regeneration process. ${ }^{2}$ Presence of requisite growth factors cause an increase in synthesis of chondrocytic matrix and causes stimulation of chondrogenous cellular proliferation. ${ }^{3}$ It causes reduction in nuclear factor kappa B activation that plays a significant function in pathogenesis of osteoarthritis by causing inhibitory action over inflammatory processes which are induced by interleukin- $1 \beta .{ }^{4}$ alpha granules found with platelets comprise of significant amounts of Growth Factors. ${ }^{5}$ Hence, application of autologous platelet rich plasma or PRP has emerged as valid 
treatment modality for osteoarthritis. It also contains plasma proteins which function similar to mesenchymal cellular adhesion molecules such as-fibrin, fibronectin, vitronectin among others. These molecules are seen during process of recovery after episode of trauma within human body. ${ }^{6}$ Platelet rich plasma or PRP has been found to contain mean of three-to-five times higher platelet numbers when compared with that in whole blood. At present, there is no widely accepted or quantified value of platelet concentrations within Platelet Rich Plasma. The numbers of platelets existing within whole blood ranges between $1,50,000$ per $\mu \mathrm{l}$ to $3,50,000$ per $\mu 1$. This maintains platelet concentration in Platelet Rich Plasma at a concentration of $200 \%$ when compared to whole blood. The concentration of platelets within platelet rich plasma varies depending upon the method of preparation which ranges between 2.5 to 8 times higher when compared to that of whole blood. Due to this, the therapeutic potential of Platelet Rich Plasma varies due to difference in techniques of preparation. ${ }^{7,8}$ The use of platelet rich plasma in treatment of degenerative osteoarthritis of knee is more safe, can be easily produced and administered with relative ease. ${ }^{9}$ It has been found to be more effective for treatment of pain and improvement in function of knee joint. Additionally, it has shown good results in treatment of early stages of osteoarthritis of knee. ${ }^{10}$ Thus, keeping all in mind all published data, this study was done to retrospective study the efficacy of intra-articular methylprednisolone therapy along with platelet rich plasma when compared with only platelet rich plasma in treatment of early osteoarthritis.

\section{METHODS}

This retrospective cross-sectional hospital-based study was designed to include 100 patients with initial stages of osteoarthritis of knee who were diagnosed as per the Kellgren-Lawrence classification. Patients were aged between 50 to 80 years. Selected patients were then categorized into; group I: It comprised of 30 subjects who were treated with intra-articular injections of platelet rich plasma in combination with methylprednisolone and group II: This group was comprised of 30 patients who were treated with intra-articular injection of platelet rich plasma alone. Complete history, clinical examination, complete blood count thrombocytopenia-related details and radiographic features were accessed from medical records of the patients who visited the Parul Institute of Medical Sciences and Research, Vadodara hospital OPD from January 2020 to March 2020. Pain levels were evaluated after treatment by using the visual analog scale (VAS) while clinical outcome was performed by using "knee injury and osteoarthritis outcome score or KOOS" at $0,1,3$, and 6 months following treatment.

\section{Selection criteria}

All patients were asked to stop use of non-steroidal antiinflammatory drugs one week prior to initiating either of the therapy protocols.

\section{Exclusion criteria}

Exclusion criteria for current study were; subjects who had been injected intra-articular injections of either steroids or hyaluronic acid in previous past year; those who had undergone arthroscopic surgery in past three months' duration; patients suffering from hematological diseases such as: anemia i.e., haemoglobin less than 7.0 $\mathrm{g} / \mathrm{dl}$, thrombocytopenia due to platelet count less than $15,000 / \mu$ litre and any bleeding dyscrasias; any bone metabolic disorder (except osteoporosis); autoimmune disorders and medical history of allergic response to steroids or any of the blood products; deformity of Valgus which is greater than $15^{\circ}$ or varus deformity which is greater than $20^{\circ}$; patients with severe ligamental instability affecting the knee joint.

\section{Platelet rich plasma preparation method}

$20 \mathrm{ml}$ of venous blood was collected from patient $(20 \mathrm{ml}$ of venous blood sample) and transferred to a test-tube which were coated with an anticoagulant (sodium citrate) for preparation of 4 to $6 \mathrm{ml}$ of platelet rich plasma containing concentration of platelets which was 3 to 6 times greater average normal parameters. The test tubes were then centrifuged at a speed of $3500 \mathrm{rpm}$ for duration of 10 minutes which separated plasma in top layer which was separated from packed red blood cells which collected at bottom layer. Collected red blood cell layer was discarded. Then, the test tubes were centrifuged for second time at $4000 \mathrm{rpm}$ for duration of 7 minutes. Thus, obtaining platelet rich plasma. Following this, approximately, 4 to $6 \mathrm{ml}$ of prepared PR product was dispensed within a sterile syringe for the purpose of injection.

\section{Technique followed for injection}

For both the study groups, intra-articular injections were given to patients in a supine position. After disinfection of overlying skin by using betadine, the medicaments were injected. $2 \mathrm{ml}$ of local anesthesthetic solution was injected first, followed by injection of platelet rich plasma or combination of PRP with methylprednisolone by using either antero-medial or antero-lateral approach. Following an observation rest period of approximately 20 minutes, patients were then instructed to perform flexing and extension of injected knees, so as to enable even spread of PRP across joint space. After this, the concerned patient was discharged.

\section{Post-injection instructions}

After receiving either of the injections, all patients were given following instructions; avoidance of running or any other high impact activities for a total duration of ten days; Ice application over the injected area for controlling pain thrice daily for approximately 10 minutes; use paracetamol as analgesic agent and avoid any NSAIDs with salicylate group throughout the follow-up period. 
Routine physical activity with low intensity in initial days, which was progressively increased till normal levels of exercise was reached.

\section{Statistical analysis}

Collected data was expressed as mean \pm standard deviation. Statistical comparison between both the groups was performed by use of Student $\mathrm{t}$-test. All calculations were performed by using the SPSS statistical software, version 21.0. Graphs were formed by using the Microsoft Excel XP version 2010.

\section{RESULTS}

\section{Demographic data}

Majority of patients who were selected for study belonged to female gender (56\%). All patient belonged to upper middle socioeconomic status.

\section{VAS scores}

VAS scores were found to decrease for both the study groups with no statistically significant difference. The differences in the VAS scores at one and three month intervals when compared with baseline scores demonstrated no statistically significant differences $(\mathrm{p}=0.08$ and 0.07$)$ between the study groups. Although, this difference was found to be of statistical significance between the groups at six months $(\mathrm{p}=0.04)$ (Table 1).

Table 1: Differences in visual analogue scale scores at baseline, 1, 3, and 6 month duration.

\begin{tabular}{|llll|}
\hline $\begin{array}{l}\text { Visual } \\
\text { analogue } \\
\text { scale }\end{array}$ & $\begin{array}{l}\text { Platelet rich plasma } \\
\text { with methyl- } \\
\text { prednisolone } \\
\text { (group I) }\end{array}$ & $\begin{array}{l}\text { Platelet } \\
\text { rich plasma } \\
\text { (group II) }\end{array}$ & $\begin{array}{l}\text { P } \\
\text { value }\end{array}$ \\
\hline Baseline & & $55.8 \pm 11.8$ & 0.67 \\
\hline Mean & $56.4 \pm 11.6$ & $36.95 \pm 17.4$ & 0.08 \\
\hline 1 month & & & \\
\hline Mean & $37.6 \pm 16.5$ & $37.23 \pm 18.6$ & 0.07 \\
\hline 3 months & & & \\
\hline Mean & $36.76 \pm 15.04$ & $41.2 \pm 13.45$ & 0.04 \\
\hline 6 months & & & \\
\hline Mean & $31.5 \pm 14.2$ & & \\
\hline
\end{tabular}

\section{KOOS outcome}

The statistical difference between both the study groups following injection at 3 months was found to be significant with respect to pain and active daily life (ADL) $(\mathrm{p}=0.01$ and 0.03 , respectively) while at the 6th month interval, it was found to be significant with respect to pain, ADL and quality of life ( $\mathrm{p}=0.001,0.02$ and 0.02 , respectively).
It was noted that differences in KOOS-QOL scores and ADL between baseline; 3 and 6 months was found to increase significantly in PRP group when compared to the methylprednisolone group. For symptoms assessment at 1 month, mean \pm SD were: $12.6 \pm 10.5$ for group I and $12.3 \pm 1.2$ for group II; three months $9.1 \pm 6.5$ for group I and $0.1 \pm 0.3$ for group II, respectively $(\mathrm{p}=0.02$ and 0.043 while at 6 months, mean \pm SD were: $23.2 \pm 7$ for group I and $13.2 \pm 2.1$ for group II $(p=0.02)$. The differences in KOOS-QOL scores and ADL scores at baseline; 3 and 6 months were found to increase significantly in PRP group than the methylprednisolone group (Table 2).

\section{DISCUSSION}

Knee is the most common of the affected joint in osteoarthritis. The main aim in managing osteoarthritis of knee joint is reduction of pain along with stiffness, to protect or regain entire range of movement and muscular strength and decreased dependence on daily activities of life. Therefore, in recent times, medications that support or improve cartilage health have been researched upon. Use of platelet rich plasma which contains growth factors are effective as well as easily available option for treatment option while treating and managing degenerative osteoarthritis. The growth factors which are released undergo binding with specific and high affinity trans-membranous receptors which leads to triggering of various intracellular signal pathways. Important growth factors that are involved are as follows- insulin-like growth factor (IGF)-1, transforming growth factor (TGF)- $\beta$, platelet-derived growth factor (PDGF), vascular endothelial growth factor (VEGF), basic fibroblast growth factor (bFGF), hepatocyte growth factor(HGF) and epidermal growth factor(EGF). Besides this, a variety of cytokines, chemokines as well as metabolites produce an additive effect on activity of these growth factors. The dense granules contained within platelets also, secrete chemicals such as-serotonin, adenosine, dopamine, calcium, histamine, adenosine diphosphate (ADP), adenosine triphosphate (ATP) and catecholamine. ${ }^{11}$ Tavassoli et al in 2019 compared clinical outcomes of treatment with platelet rich plasma and hyaluronic acid injections in patients who were diagnosed with bilateral osteoarthritis affecting the knee joint in a randomized control trial by using the Western Ontario and McMaster Universities arthritis index (WOMAC). The pain subscale was seen to show extremely significant $\mathrm{p}$ values from baseline to $12^{\text {th }}$ week of intervention on comparing platelet rich plasma and hyaluronic acid. Greatest efficaciousness of platelet rich plasma was noted in the $4^{\text {th }}$ week with approximately $50 \%$ reduction in the symptoms compared with $25 \%$ decrease in patients treated with hyaluronic acid. ${ }^{12}$ Kurapati et al in their combination therapy following use of both platelet rich plasma and hyaluronic acid on patients suffering from initial stage of knee osteoarthritis reported statistically significant improvement in VAS scores. Significant improvement from values at $3.00 \pm 0.49$ recorded at baseline level to $1.57 \pm 0.41$ in grade $\mathrm{I}(\mathrm{p}=0.031)$ and 
Table 2: Differences in knee injury and osteoarthritis outcome scores in both the study groups following injections at baseline, 1, 3 and 6 months.

\begin{tabular}{|c|c|c|c|c|c|c|c|c|}
\hline KOOS & Baseline & P value & 1 month & P value & 3 months & $P$ value & 6 months & P value \\
\hline \multicolumn{9}{|l|}{ Pain $($ mean \pm SD) } \\
\hline PRP+methylprednisolone & $43.7 \pm 11.3$ & \multirow{2}{*}{0.56} & $46.3 \pm 19$ & \multirow{2}{*}{0.19} & $42.7 \pm 12.1$ & \multirow{2}{*}{0.01} & $44.3 \pm 19.3$ & \multirow{2}{*}{0.01} \\
\hline Methylprednisolone & $44.7 \pm 13.4$ & & $53.8 \pm 13.9$ & & $39.3 \pm 11.4$ & & $40.1 \pm 10.7$ & \\
\hline \multicolumn{9}{|l|}{ Symptoms $($ mean \pm SD) } \\
\hline PRP+methylprednisolone & $32.4 \pm 11.1$ & \multirow{2}{*}{0.35} & $12.6 \pm 10.5$ & \multirow{2}{*}{0.02} & $12.3 \pm 1.2$ & \multirow{2}{*}{0.043} & $13.2 \pm 7$ & \multirow{2}{*}{0.04} \\
\hline Methylprednisolone & $31.0 \pm 17.5$ & & $12.3 \pm 1.2$ & & $0.1 \pm 0.3$ & & $0.2 \pm 0.1$ & \\
\hline \multicolumn{9}{|c|}{ Activities of daily living (mean \pm SD) } \\
\hline PRP+methylprednisolone & $36.1 \pm 15.3$ & \multirow{2}{*}{0.1} & $44.7 \pm 18.6$ & \multirow{2}{*}{0.2} & $49.7 \pm 18.8$ & \multirow{2}{*}{0.02} & $49.5 \pm 12.6$ & \multirow{2}{*}{0.02} \\
\hline Methylprednisolone & $35.8 \pm 15.8$ & & $51.6 \pm 15.8$ & & $47.3 \pm 14.3$ & & $48.0 \pm 13.5$ & \\
\hline \multicolumn{9}{|c|}{ Sport/recreation $($ mean \pm SD) } \\
\hline PRP+methylprednisolone & $21.1 \pm 10.1$ & \multirow{2}{*}{0.6} & $30.5 \pm 21.5$ & \multirow{2}{*}{0.4} & $33.2 \pm 21.1$ & \multirow{2}{*}{0.19} & $33.1 \pm 20.5$ & \multirow{2}{*}{0.18} \\
\hline Methylprednisolone & $19.2 \pm 10.0$ & & $29.1 \pm 20.0$ & & $31.2 \pm 12.1$ & & $30.1 \pm 11.2$ & \\
\hline \multicolumn{9}{|c|}{ Quality of life $($ mean \pm SD $)$} \\
\hline PRP+methylprednisolone & $21.2 \pm 10.8$ & \multirow{2}{*}{0.19} & $23.9 \pm 11.3$ & \multirow{2}{*}{0.17} & $29.9 \pm 22.9$ & \multirow{2}{*}{0.15} & $30.1 \pm 20.1$ & \multirow{2}{*}{0.12} \\
\hline Methylprednisolone & $23.1 \pm 12.3$ & & $24.2 \pm 13.1$ & & $26.2 \pm 11.2$ & & $25.2 \pm 11.1$ & \\
\hline
\end{tabular}

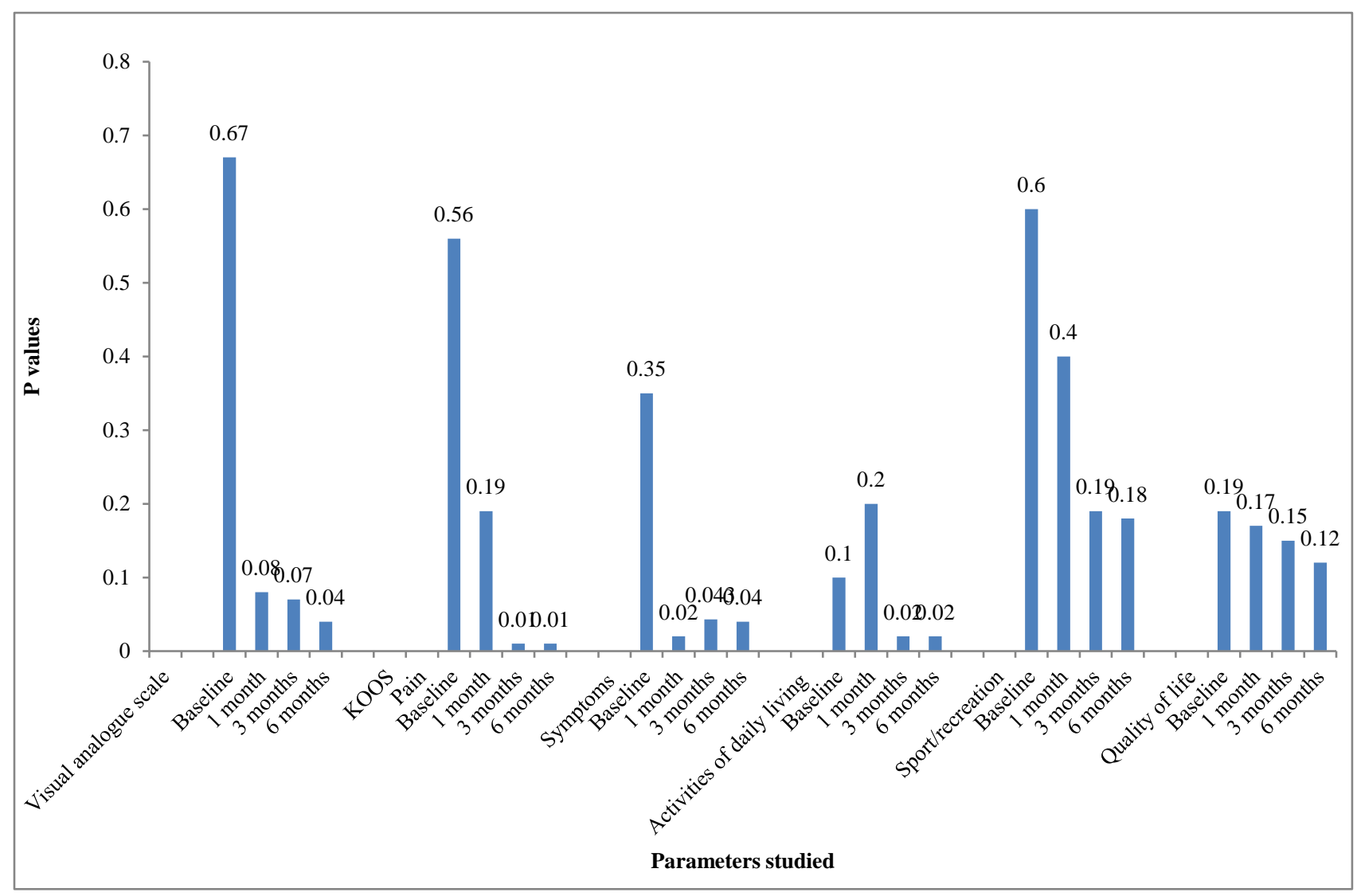

Figure 1: P values for VAS and KOOS.

Ismaiel in 2018 compared effects of intra-articular injections of platelet-rich plasma and corticosteroid in causing reduction of pain and improvement in function. Statistically significant differences between both the study groups were observed. Also, greater degree of improvement was seen in the study group treated with platelet rich plasma. ${ }^{14}$ Forogh et al showed significant improvement in VAS pain score, 20 minute walking test and in entire subcategories of the KOOS questionnaire (i.e., relief in pain, activity of daily living/ADL, 
symptoms and quality of life). ${ }^{15}$ Calis et al evaluated the effectiveness of autologous platelet- rich plasma therapy on pain, functional status and cartilaginous regeneration in advanced osteoarthritis affecting knee. While comparing the mean visual analogue scale values before any treatment, it was noted that these values had undergone significant decrease $(p<0.001)$ after third and sixth months of treatment. Significant increase $(p<0.05)$ in cartilage thicknesses was observed following treatment at 3rd and 6th months when compared with values before initiating treatment. ${ }^{16}$ Halpern et al reported a significant decrease in VAS pain score as well as WOMAC pain score on follow-up at 6th month and 1 year intervals, respectively in patients suffering from osteoarthritis of knee. ${ }^{17}$ Gobbi et al in their study on Platelet Rich Plasma showed significant Improvements in pain scores and functionality at six and twelve months intervals. ${ }^{18}$ Present study showed that treatment of initial stages of osteoarthritis can be treated more effectively by intraarticular injections of platelet rich plasma combined with methylprednisolone when compared with Platelet rich plasma alone. It is demonstrated by progressive increase in statistically significance on follow-up at 1 month, 3 momthsand 6months intervals using the KOOS (knee injury and osteoarthritis outcome score) and visual analogue scale (VAS) score values.

\section{CONCLUSION}

Treatment of osteoarthritis of knee joint in initial stages has not been studied in detail though there are studies related to treatment in advanced stages. Our study has analyzed combination therapy using PRP and methylprednisolone than compared to PRP alone. We have concluded that using this combination can effectively reduce symptoms, improve an individual's daily life activities and quality of life. Larger randomized controlled trials are required for further elaborative study on this combination therapy and generation of elaborate data which may be useful in treating such cases.

\section{ACKNOWLEDGEMENTS}

Authors would like to thank Dr. Niraj Jain, Dr. Alok Pandey, for his support during study.

Funding: No funding sources Conflict of interest: None declared

Ethical approval: The study was approved by the Institutional Ethics Committee

\section{REFERENCES}

1. Felson DT. Clinical practice. Osteoarthritis of the knee. N Engl J Med. 2006;354:841-8.

2. Handl M, Amler E, Bräun K, Holzheu J, Trc T, Imhoff $A B$, et al. Positive effect of oral supplementation with glycosaminoglycans and antioxidants on the regeneration of osteochondral defects in the knee joint. Physiol Res. 2007;56:2439.

3. Fortier LA, Barker JU, Strauss EJ, McCarrel TM, Cole BJ. The role of growth factors in cartilage repair. Clin Orthop Relat Res. 2011;469:2706-15.

4. van Buul GM, Koevoet WL, Kops N, Bos PK, Verhaar JA, Weinans $\mathrm{H}$, et al. Platelet-rich plasma release inhibits inflammatory processes in osteoarthritic chondrocytes. Am J Sports Med. 2011; 39:2362-70.

5. Cook JL, Anderson CC, Kreeger JM, Tomlinson JL. Effects of human recombinant interleukin-1beta on canine articular chondrocytes in three-dimensional culture. Am J Vet Res. 2000;61:766-70.

6. Kang YH, Jeon SH, Park JY, Chung JH, Choung $\mathrm{YH}$, Choung $\mathrm{HW}$, et al. Platelet rich fibrin is a Bioscaffold and reservoir of growth factors for tissue regeneration. Tissue Eng. 2011;17:349-59.

7. Marx RE. Platelet-rich plasma (PRP): what is PRP and what is not PRP?. Implant Dent. 2001;10:225-8.

8. de Mos M, van der Windt AE, Jahr H, van Schie HT, Weinans H, Verhaar JA, et al. Can platelet-rich plasma enhance tendon repair? A cell culture study. Am J Sports Med. 2008;36:1171-8.

9. Forogh B, Mianehsaz E, Shoaee S, Ahadi T, Raissi GR, Sajadi S. Effect of single injection of plateletrich plasma in comparison with corticosteroid on knee osteoarthritis: a double-blind randomized clinical trial. J Sports Med Phys Fitness. 2016;56: 901-8.

10. Richmond J, Hunter D, Irrgang J. Treatment of osteoarthritis of the knee (non arthroplasty). J Am Acad Orthop Surg. 2009; 17:591-600.

11. Navani A, Li G, Chrystal J. Platelet rich plasma in musculoskeletal pathology: A necessary rescue or lost cause? Pain Physician. 2017;20:E345-56.

12. Tavassoli M, Janmohammadi N, Hosseini A, Khafri S, Esmaeilnejad-Ganji SM. Single- and double-dose of platelet-rich plasma versus hyaluronic acid for treatment of knee osteoarthritis: A randomized controlled trial. World J Orthop. 2019;10(9:310-26.

13. Kurapati K, Tapadia S, Rao M, Anbarasu K, Verma VK, Beevi SS. Efficacy of Intra-articular Injection of Platelet Rich Plasma and Hyaluronic Acid in Early Knee Osteoarthritis - Case Series. Eur J Mol Clin Med. 2018;5(1):30-6.

14. Ismaiel AH. Comparison between the effect of intra-articular injections of platelet-rich plasma and corticosteroids in advanced knee osteoarthritis. J Med Sci Res. 2018;1:278-84.

15. Forogh B, Mianehsaz E, Shoaee S, Ahadi T, Raissi GR, Sajadi S. Effect of single injection of plateletrich plasma in comparison with corticosteroid on knee osteoarthritis: a double-blind randomized clinical trial. J Sports Med Phys Fitness. 2016;56(78):901-8.

16. Calis HT, Sutbeyaz ST, Guler E, Halici C, Sayan H, Koc A, et al. Efficacy of intra-articular platelet rich application in knee osteoarthritis. Arch Rheumatol. 2015;30(3):198-205. 
17. Halpern B, Chaudhury S, Rodeo SA, et al. Clinical and MRI outcomes after platelet-rich plasma treatment for knee osteoarthritis. Clin J Sport Med. 2013;23(03):238-9.

18. Gobbi A, Karnatzikos G, Mahajan V, Malchira S. Platelet-rich plasma treatment in symptomatic patients with knee osteoarthritis: preliminary results in a group of active patients. Sports Health. 2012; 4(02):162-72.
Cite this article as: Mahajan KK, Dudhia SH.

Efficacy of intra-articular methylprednisolone along with platelet rich plasma vs. platelet rich plasma in early osteoarthritis: a retrospective study. Int J Basic Clin Pharmacol 2021;10:1265-70. 